\title{
Numerical Chebyshev Approximation by Interpolating Rationals
}

\author{
By Jack Williams
}

\begin{abstract}
The paper is concerned with the Chebyshev approximation of decay-type functions $f(x)$ by interpolating rationals. The interpolating points are chosen to be the zeros of $f(x)$. Existence, uniqueness and characterization of best approximations are first shown. An exchange algorithm is then described for computing the best approximation.
\end{abstract}

1. Introduction. A common problem in various branches of physics and chemistry is the approximation of oscillating decay-type functions. Denote the class of such continuous functions by $D[0, b], 0<b<\infty$, where $f \in D[0, b]$ is of the form $f=B(x) g(x), g \in C[0, b], g>0 \forall x \in[0, b]$, and $B(x)$ is the "oscillation" factor satisfying $B\left(x_{v}\right)=0$ for distinct $x_{v} \in[0, b], \nu=1,2, \cdots, R$. In practice, $f$ is frequently defined numerically so that $B(x)$ may conveniently be chosen to be a polynomial factor constructed from the approximated zeros of $f(x)$.

In this paper, a numerical method is presented for computing Chebyshev approximations of $f \in D[0, b]$ in the form of interpolating rationals. Existence, uniqueness and characterization of best approximations are first established; an extended form of the exchange algorithm is then obtained for computing the best approximation. Some simple numerical examples are also presented.

2. The Class of Approximating Functions. Given $f \in D[0, b]$, let $V$ denote the class of approximating functions

$$
F(A, x)=\frac{B(x)}{(L(A, x))^{p}}, \quad x \in[0, b]
$$

which satisfy

(a) $L(A, x)=\sum_{r=1}^{n} a_{r} \phi_{r}(x), \phi_{1} \equiv 1, \phi_{r} \in C[0, b], r=2,3, \cdots, n$, where $\left\{\phi_{1}, \phi_{2}, \cdots, \phi_{n}\right\}$ forms a Chebyshev set on $[0, b]$

(b). The parameter space $P$ consists of the points $A=\left(a_{1}, a_{2}, \cdots, a_{n}\right)$ for which $L(A, x)>0, \forall x \in[0, b]$

(c) $p>0$.

The form of the approximating functions is justified by the following trivial application of Weierstrass' theorem.

LEMMA 2.1. Given $f \in D[0, b]$, let $V$ denote the above class of approximating functions where $L(A, x)=\sum_{r=1}^{n} a_{r} x^{r-1}$, then $f$ is uniformly approximable by elements of $V$.

The practical effectiveness of approximating functions of this form is moreover strongly suggested by the efficiency of approximations such as those of Hastings [3] for the negative exponential function.

Received January 8, 1971, revised May 10, 1971.

AMS 1970 subject classifications. Primary 65D15, 65D20.

Key words and phrases. Chebyshev approximation, exchange algorithm.

Copyright $\odot$ 1972, American Mathematical Society 
Let $\|\cdot\|$ denote the Chebyshev norm on $[0, b]$, then $F\left(A^{*}, x\right) \in V$ is a best Chebyshev approximation of $f$ if $\left\|f-F\left(A^{*}, x\right)\right\| \leqq\|f-F(A, x)\|, \forall F(A, x) \in V$.

3. Existence, Uniqueness and Characterization.

THEOREM 3.1. Let $f \in D[0, b], f \notin V$, then there exists a best approximation $F\left(A^{*}, x\right)$ $\in V$ of $f$.

Proof. For each $F(A, x)=B(x) g(x) \in V, B(x)$ is a fixed function and satisfies $B\left(x_{v}\right)=0, x_{v} \in[0, b], \nu=1,2, \cdots, R$. A contender for a best approximation is

$$
F\left(A_{0}, x\right)=B(x) / M^{-1}, \text { where } 0<M<2 \cdot \min _{0 \leq x \leq b}(g(x)) \text {. }
$$

Given small $\delta>0$, let $O_{\nu}:=\left\{x:\left|x-x_{\nu}\right|<\delta\right\}, 1 \leqq \nu \leqq R$, and $P_{\delta}=\bigcup_{\nu-1}^{R} O_{\nu}$, then since $|B(x)| \neq 0$ in $[0, b]-P_{\delta},|B(x) g(x)-M \cdot B(x)|<|B(x) g(x)|, x \in[0, b]-P_{\delta}$. Therefore, by continuity and compactness, $\exists h>0$ (not necessarily small) satisfying

$$
\left|f(x)-F\left(A_{0}, x\right)\right| \leqq|f(x)|-h \leqq\|f\|-h, \quad x \in[0, b]-P_{8} .
$$

Also, by continuity it is possible to choose $\delta$ sufficiently small, so that

$$
\left|f(x)-F\left(A_{0}, x\right)\right|<\|f\|-h, \quad x \in O_{v}, 1 \leqq \nu \leqq R,
$$

giving $\left\|f-F\left(A_{0}, x\right)\right\| \leqq\|f\|-h$. Clearly a best approximation, if one exists, must lie in the parameter set

$$
P_{M}:=\{A:\|f-F(A, x)\| \leqq\|f\|-h\} .
$$

Now, there exists a sequence of parameters $\left\{A_{k}\right\}$ in $P_{M}$ for which

$$
\lim _{k \rightarrow \infty}\left\|f-F\left(A_{k}, x\right)\right\|=\inf _{A \in P_{M}}\|f-F(A, x)\| .
$$

For each $A \in P_{M},\|F(A, x)\| \geqq h$, so there exists an interval $[c, d] \subset[0, b]$ for which $|F(A, x)| \geqq h / 2, x \in[c, d]$.

Hence, $|L(A, x)| \leqq\{2|B(x)| / h\}^{1 / p}, x \in[c, d]$, and it follows that $A$ is bounded [5, p. 24]. The sequence of parameters $A_{k}=\left(a_{1}^{(k)}, a_{2}^{(k)}, \cdots, a_{n}^{(k)}\right)$ in (3.1) therefore satisfy $\left|a_{r}^{(k)}\right| \leqq M_{1}$, for all $k, 1 \leqq r \leqq n$; so there exists a subsequence of $\left\{A_{k}\right\}$ which converges to $A^{*}$ and existence is proved.

Characterization and uniqueness of best approximations may be established by applying the theory of Meinardus and Schwedt, which is conveniently presented in [4]. Their theory introduces the notion of asymptotic convexity and we state their results here as applied to real-valued functions on a compact subset $X$ of the real line.

Definition [4, p. 136]. A set $V$ of functions $F(A, x), x \in X$, is called asymptotically convex provided that for each pair of elements $A$ and $B$ in the parameter space $P$ and each real $t, 0 \leqq t \leqq 1$, their exists $A(t) \in P$ and a continuous function $g(x, t)$ on $X \times[0,1]$ with $g(x, 0)>0$ such that

$$
\|(1-\operatorname{tg}(x, t)) F(A, x)+\operatorname{tg}(x, t) F(B, x)-F(A(t), x)\|=o(t) \text { as } t \rightarrow 0 .
$$

Remark. Let $h \in C[X]$, which may or may not vanish at a finite number of points in $X$, then the set of functions $h(x) F(A, x), F(A, x) \in V$, is also asymptotically convex.

THEOREM $3.2\left[4\right.$, p. 138]. Let $V$ be asymptotically convex, then $F\left(A^{*}, x\right) \in V$ is a best approximation of $f \in C[X]$ if and only if 


$$
\min _{x \in X^{*}}\left\{\left(f(x)-F\left(A^{*}, x\right)\right)\left(F(B, x)-F\left(A^{*}, x\right)\right)\right\} \leqq 0
$$

is satisfied $\forall B \in P$ where

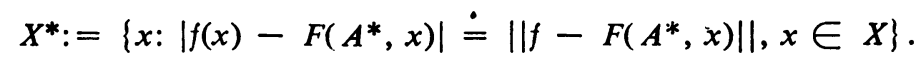

Consider now the application of these results to the class of functions $V$ of (2.1). From the above remark, it is sufficient to show that the functions $1 /(L(A, x))^{p}$ are asymptotically convex; this follows trivially from a simple method of Meinardus and Schwedt [4, p. 139]. By hypothesis, the extremal set $X^{*}$ in Theorem 3.2 cannot contain points $x, \in[0, b]$ at which $B\left(x_{v}\right)=0$. Hence, $F\left(A^{*}, x\right) \in V$ is a best approximation of $f \in D[0, b]$ if and only if

$$
\min _{x \in X^{*}}\left\{\left(g(x)-L\left(A^{*}, x\right)^{-D}\right)\left(L(B, x)^{-p}-L\left(A^{*}, x\right)^{-D}\right)\right\} \leqq 0, \quad \forall B \in P .
$$

Employing the Chebyshev set hypothesis on $\left\{\phi_{r}\right\}$ gives

THEOREM 3.3. $F\left(A^{*}, x\right) \in V$ is a best approximation of $f \in D[0, b]$ if and only if there exist points $x_{r} \in[0, b], 1 \leqq r \leqq n+1$, with $0 \leqq x_{1}<x_{2}<\cdots<x_{n+1} \leqq b$ such that

$$
\left|f\left(x_{r}\right)-F\left(A^{*}, x_{r}\right)\right|=\left\|f-F\left(A^{*}, x_{r}\right)\right\|, \quad 1 \leqq r \leqq n+1,
$$

and

$$
\begin{array}{r}
{\left[f\left(x_{r}\right)-F\left(A^{*}, x_{r}\right)\right] \operatorname{sign}\left[f\left(x_{r}\right)\right]=-\left[f\left(x_{r+1}\right)-F\left(A^{*}, x_{r+1}\right)\right] \operatorname{sign}\left[f\left(x_{r+1}\right)\right],} \\
1 \leqq r \leqq n .
\end{array}
$$

In addition, we have the following useful generalization of a theorem of de la Vallée Poussin (see [4, p. 82]).

THEOREM 3.4. Let $F\left(A^{*}, x\right)$ be a best approximation of $f \in D[0, b]$. Let there exist $n+1$ points $x_{r} \in[0, b], x_{r}<x_{r+1}$, which satisfy, for a given $F(A, x) \in V$,

$$
f\left(x_{r}\right)-F\left(A, x_{r}\right) \neq 0, \quad 1 \leqq r \leqq n+1,
$$

and

$$
\operatorname{sign}\left(S_{r}\right)=-\operatorname{sign}\left(S_{r+1}\right), \quad 1 \leqq r \leqq n,
$$

where $S_{r}=\operatorname{sign}\left(f\left(x_{r}\right)\right) \cdot\left[f\left(x_{r}\right)-F\left(A, x_{r}\right)\right], 1 \leqq r \leqq n+1$. Then,

$$
\min _{1 \leq r \leq n+1}\left|f\left(x_{r}\right)-F\left(A, x_{r}\right)\right| \leqq\left\|f-F\left(A^{*}, x_{r}\right)\right\| \leqq\|f-F(A, x)\| .
$$

The lower bound is easily established (using the Chebyshev set hypothesis) with the aid of a general theorem of Meinardus and Schwedt [4, p. 134]. A further useful result from their theorem is

COROLlaRY. Let the conditions of Theorem 3.4 be satisfied and let $\left|f\left(x_{r}\right)-F\left(A, x_{r}\right)\right|$ $=\left|f\left(x_{r+1}\right)-F\left(A, x_{r+1}\right)\right|, 1 \leqq r \leqq n$, then $F(A, x)$ is a best approximation of $f$ on the point set $\left\{x_{r}\right\}$ with respect to the approximating functions $V$.

Having established characterization, uniqueness follows by a slightly modified form (taking account of the extra zeros in $B(x)$ ) of a theorem of Meinardus and Schwedt [4, p. 146]. Full details are given in [6].

4. The Numerical Method. The above results form the basis for the application 
of an exchange algorithm. An initial reference $X^{(0)}:=\left\{x_{1}^{(0)}, x_{2}^{(0)}, \cdots, x_{n+1}^{(0)}\right\} \subset[0, b]$ is selected and the $k$ th iteration of the algorithm consists of the following two-stage process.

Stage I. An approximation $F\left(A_{k}, x\right) \in V$ is calculated from

$$
E_{k}\left(x_{r}^{(k)}\right)=\left|B\left(x_{r}^{(k)}\right)\right|\left\{g\left(x_{r}^{(k)}\right)-L\left(A_{k}, x_{r}^{(k)}\right)^{-p}\right\}=(-)^{r} \lambda_{k}, 1 \leqq r \leqq n+1 .
$$

It follows from the corollary of Theorem 3.4 that $F\left(A_{k}, x\right)$ is a best approximation (w.r.t. $V$ ) of $f$ on $X^{(k)}$.

Stage II. The extrema of the error function $E_{k}(x)$ are found from which a new reference $X^{(k+1)}$ is selected, satisfying

(a) $E_{k}(x)$ alternates in sign on $X^{(k+1)}$,

(b) $\min _{x \in X(k+1)}\left|E_{k}(x)\right| \geqq\left|\lambda_{k}\right|$.

In particular, $X^{(k+1)}$ will always contain a point at which $\left|E_{k}(x)\right|$ attains its maximum value on $[0, b]$.

Under these conditions, Theorem 3.4 and the Corollary give $\left|\lambda_{k}\right|<\left|\lambda_{k+1}\right|$ (assuming $F\left(A_{k}, x\right)$ is not the best approximation). It is now possible, using Theorem 3.3, to show that the sequence $\left\{F\left(A_{k}, x\right)\right\}$ converges uniformly to the best approximation $F\left(A^{*}, x\right)$. The proof is exactly the same as Rice's proof [5, p. 178] for unisolvent functions, except of course, for the actual form of characterization used. Concerning the first stage of the algorithm, we have

THEOREM 4.1. For an arbitrary reference $X:=\left\{x_{r}\right\}, B\left(x_{r}\right) \neq 0,1 \leqq r \leqq n+1$, there exists a unique real solution of Eqs. (4.1).

Proof. From (4.1),

$$
\sum_{i=1}^{n} a_{i} \phi_{i}\left(x_{r}\right)-\alpha_{r}(\lambda)=0, \quad 1 \leqq r \leqq n+1,
$$

where $\alpha_{r}(\lambda)=\left\{\left|B\left(x_{r}\right)\right| /\left(\left|B\left(x_{r}\right)\right| g\left(x_{r}\right)-(-)^{r} \lambda\right)\right\}^{1 / p}$. We consider the parameter set $(A, \lambda)$, where $\lambda \in\left(-\rho_{1}, \rho_{2}\right)$,

$$
\rho_{1}=\min _{r \geq 1}\left\{\left|B\left(x_{2 r-1}\right)\right| g\left(x_{2 r-1}\right)\right\}, \quad \rho_{2}=\min _{r \geq 1}\left\{\left|B\left(x_{2 r}\right)\right| g\left(x_{2 r}\right)\right\} .
$$

Eliminating $A$ from (4.2), the equations reduce to

$$
\left.F(\lambda)=\sum_{r=1}^{n+1} \mu_{r} \alpha_{r} \dot{\lambda}\right)=0,
$$

where, using properties of Chebyshev sets, we may choose $\left\{\mu_{r}\right\}$ such that $\operatorname{sign}\left(\mu_{r}\right)=$ $(-)^{r}, 1 \leqq r \leqq n+1$. Now, $F(\lambda)$ is differentiable on $\left(-\rho_{1}, \rho_{2}\right)$ and

$$
\alpha_{r}^{\prime}(\lambda)=\frac{(-)^{r}}{p\left|B\left(x_{r}\right)\right|}\left\{\frac{\left|B\left(x_{r}\right)\right|}{\left|B\left(x_{r}\right)\right| g\left(x_{r}\right)-(-)^{r} \lambda}\right\}^{(1 / p+1)},
$$

hence, $F^{\prime}(\lambda)>0, \lambda \in\left(-\rho_{1}, \rho_{2}\right)$. Since $\lim _{\lambda+\rightarrow-\rho_{1}} F(\lambda)=-\infty, \lim _{\lambda \rightarrow \rho_{2}} F(\lambda) \rightarrow+\infty$, there exists a unique $\lambda^{*} \in\left(-\rho_{1}, \rho_{2}\right)$ with $F\left(\lambda^{*}\right)=0$.

The Newton iteration was found to be completely successful for the numerical solution of (4.1). However, for the convergence of the exchange algorithm we must in practice check that at each step $F\left(A_{k}, x\right) \in V$. 
The extrema search of Stage II is slightly complicated by the extra extrema of $E_{k}(x)$ introduced by the factor $|B(x)|$. Thus, special care is required when searching for an extremum in the neighborhood of an interior zero of $f(x)$. Here, in general, $E_{k}(x)$ will have two extrema of the same sign and two local searches are required (at least for the first iteration). This is illustrated by a simple example in Fig. 1.

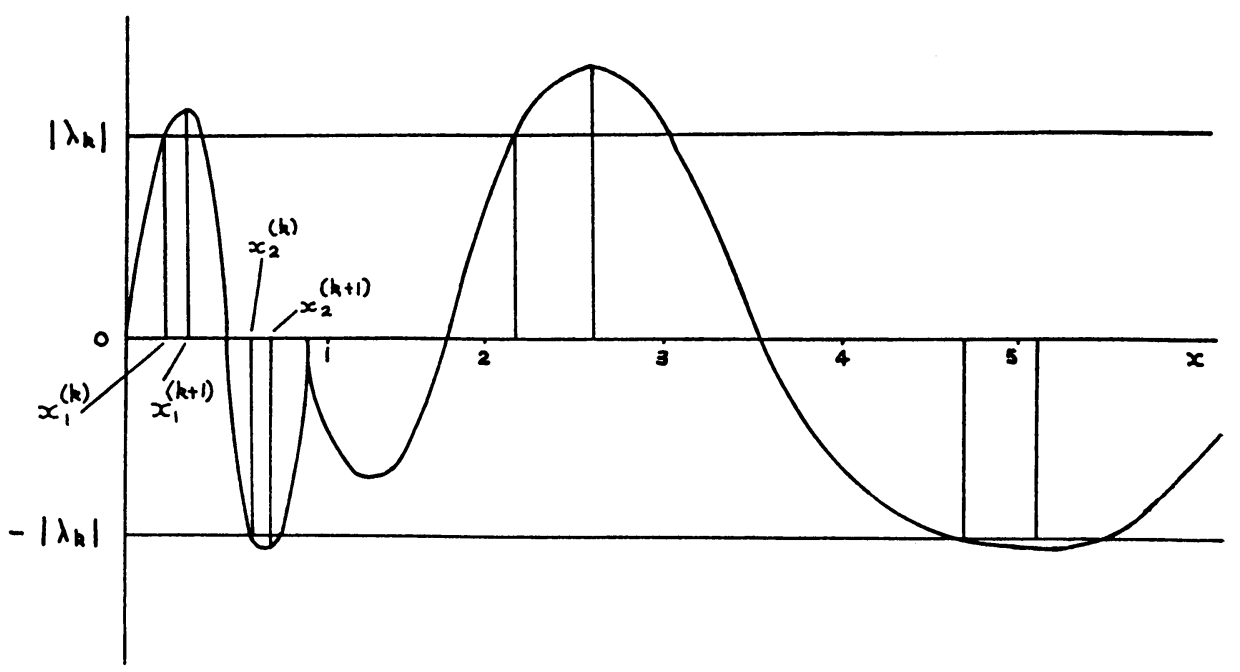

FIGURE 1. Typical error curve, $n=3, R=2$.

Apart from this complication, the extrema search considerations are well known [2].

Naturally, the success of the algorithm depends to a large extent on the choice of the initial reference $X^{(0)}$. We found the most convenient method for dealing with this problem was to follow the approach of Appel [1] and obtain a linearized discrete least squares approximation of $f(x)$. With $f(x)=F(A, x)+\eta(x)$ we have linearized equations

$$
\sum_{r=1}^{n} a_{r} \phi_{r}\left(x_{k}\right)+\tilde{\eta}\left(x_{k}\right)=\left[\frac{B\left(x_{k}\right)}{f\left(x_{k}\right)}\right]^{1 / \nu} \equiv G\left(x_{k}\right), \quad 1 \leqq k \leqq N .
$$

where $\eta(x)=-p(f(x) / G(x)) \tilde{\eta}(x)+O\left(\eta^{2}\right), N>n$. The weighted least squares problem $\sum_{k=1}^{P} w_{k} \tilde{\eta}\left(x_{k}\right)^{2}$ can now be solved with the aid of orthogonal polynomials. It follows that the resulting $\tilde{F}(A, x)$ yields an error function $E(x)$ with the appropriate sign alternation properties. Therefore, if $\widetilde{F}(A, x) \in V$, it may be used to obtain $X^{(0)}$ and provide starting values for the Newton iteration of Stage I.

5. Numerical Examples. (i) Several forms of approximations were successfully obtained for decay-type functions defined numerically. Typically, two examples $f_{3 p}$ and $f_{3}$, are given in Fig. 2 for which approximations of the form 


$$
\frac{x^{2}(\alpha-x)}{\left(\sum_{r=0}^{6} a_{r} x^{r}\right)^{2}} \text { and } \frac{x\left(\alpha_{1}-x\right)\left(\alpha_{2}-x\right)}{\left(\sum_{r=0}^{6} a_{r} x^{r}\right)^{2}} \text {, }
$$

respectively, were found to be the most satisfactory. These produced errors of order $10^{-4}$ (valid on $[0, \infty]$ ) and required three iterations of the exchange algorithm.
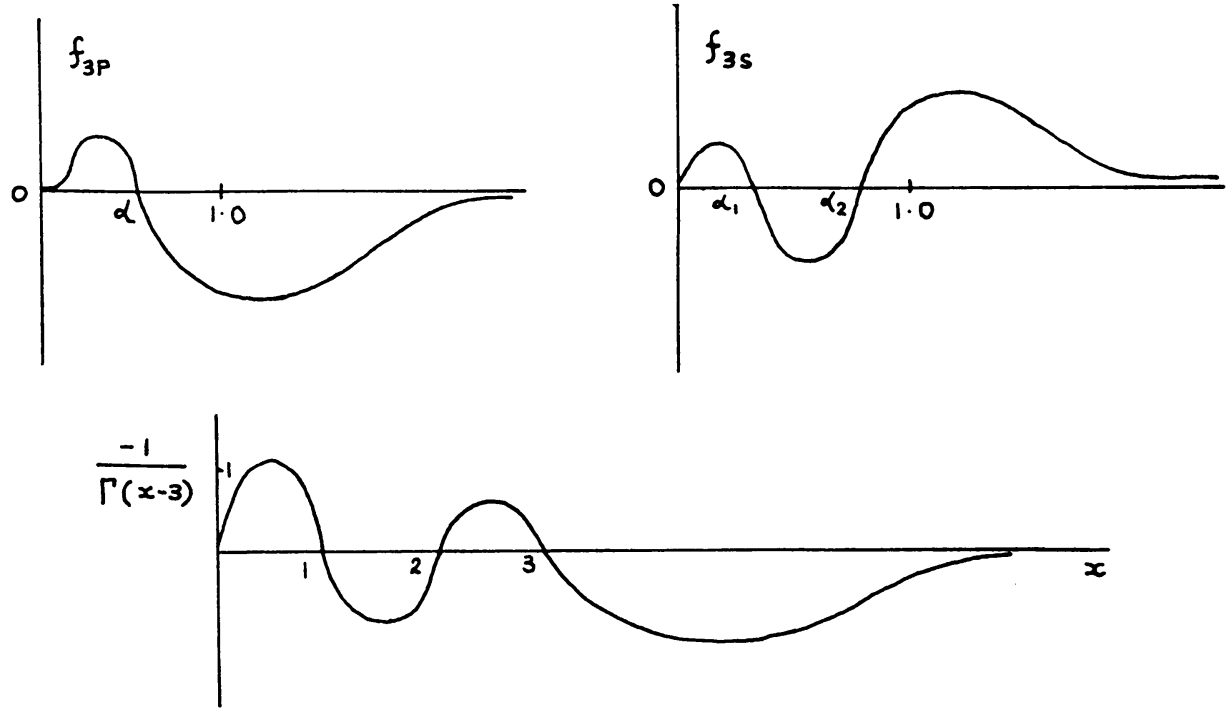

FIGURE 2

(ii) $f(x)=10 x^{2}(1-x) e^{-2 x}$. We considered approximations of the form

$$
F(A, x)=x^{2}(1-x) /\left(\sum_{r=0}^{n} a_{r} x^{r}\right)^{p}
$$

and obtained the results given in Table 1 in which the effectiveness of the "least TABLE 1

\begin{tabular}{rrccc}
\multicolumn{5}{c}{ Approximation of $f(x)=10 x^{2}(1-x) e^{-2 x}$} \\
\hline$n$ & $p$ & $\min _{1 \leq i \leq n+2}\left|E_{0}\left(x_{i}\right)\right|$ & $\left\|E_{0}\right\|$ & $\left\|f-F\left(A^{*}, x\right)\right\|$ \\
\hline 4 & 2 & $0.3 \cdot 10^{-2}$ & $2.0 \cdot 10^{-2}$ & $1.26 \cdot 10^{-2}$ \\
& 4 & $0.4 \cdot 10^{-3}$ & $2.0 \cdot 10^{-3}$ & $1.25 \cdot 10^{-3}$ \\
& 6 & $1.0 \cdot 10^{-4}$ & $4.5 \cdot 10^{-4}$ & $2.92 \cdot 10^{-4}$ \\
& 8 & $0.4 \cdot 10^{-4}$ & $1.5 \cdot 10^{-4}$ & $1.01 \cdot 10^{-4}$ \\
& 10 & $1.8 \cdot 10^{-5}$ & $6.6 \cdot 10^{-5}$ & $4.35 \cdot 10^{-5}$ \\
\hline 6 & 2 & $0.2 \cdot 10^{-3}$ & $1.5 \cdot 10^{-3}$ & $8.78 \cdot 10^{-4}$ \\
& 4 & $1.0 \cdot 10^{-5}$ & $4.3 \cdot 10^{-5}$ & $2.62 \cdot 10^{-5}$ \\
& 6 & $1.1 \cdot 10^{-6}$ & $4.7 \cdot 10^{-6}$ & $3.01 \cdot 10^{-6}$ \\
& 8 & $2.2 \cdot 10^{-7}$ & $9.5 \cdot 10^{-7}$ & $6.15 \cdot 10^{-7}$ \\
& 10 & $0.6 \cdot 10^{-7}$ & $2.7 \cdot 10^{-7}$ & $1.75 \cdot 10^{-7}$ \\
\hline 8 & 2 & $1.3 \cdot 10^{-5}$ & $9.1 \cdot 10^{-5}$ & $5.48 \cdot 10^{-5}$ \\
& 4 & $1.5 \cdot 10^{-7}$ & $8.9 \cdot 10^{-7}$ & $5.54 \cdot 10^{-7}$ \\
& 6 & $1.0 \cdot 10^{-8}$ & $4.9 \cdot 10^{-8}$ & $2.98 \cdot 10^{-8}$ \\
& 8 & $1.2 \cdot 10^{-9}$ & $5.7 \cdot 10^{-9}$ & $3.51 \cdot 10^{-9}$ \\
& 10 & $0.2 \cdot 10^{-9}$ & $1.1 \cdot 10^{-9}$ & $6.47 \cdot 10^{-10}$ \\
\hline
\end{tabular}


squares" starting values is shown by the inclusion interval of Theorem 3.4,

$$
\min _{1 \leq i \leq n+2}\left|E_{0}\left(x_{i}\right)\right| \leqq\left\|f-F\left(A^{*}, x\right)\right\| \leqq\left\|E_{0}\right\| .
$$

All the approximations are valid on $[0, \infty]$. The progress of the exchange algorithm is shown in Table 2 for the case $n=6, p=8$ where each row corresponds to a ref-

TABLE 2

The Exchange Algorithm

\begin{tabular}{ccccc}
\hline$x_{1}$ & 0.1218 & 0.1387 & 0.1404 & 0.1404 \\
$x_{2}$ & 0.3835 & 0.4592 & 0.4487 & 0.4472 \\
$x_{3}$ & 1.2806 & 1.3258 & 1.3342 & 1.3349 \\
$x_{4}$ & 1.9369 & 2.0420 & 2.0602 & 2.0607 \\
$x_{5}$ & 2.7843 & 2.9470 & 2.9557 & 2.9555 \\
$x_{6}$ & 3.8788 & 4.1124 & 4.1070 & 4.1068 \\
$x_{7}$ & 5.3619 & 5.6805 & 5.6774 & 5.6772 \\
$x_{8}$ & 7.8190 & 8.1613 & 8.1592 & 8.1589 \\
\hline$\lambda \cdot 10^{7}$ & -5.6845 & -6.1480 & -6.1510 & -6.1511 \\
\hline
\end{tabular}

erence point and each column to an iteration.

(iii) $f(x)=-1 / \Gamma(x-3)$. For approximation by

$$
F(A, x)=\frac{x(1-x)(2-x)(3-x)}{\left(\sum_{r=0}^{n} a_{r} x^{r}\right)^{p}},
$$

the value $p=4$ was found to be the most satisfactory. The resulting approximations with errors shown in Table 3 are valid on $[0, \infty]$ and required four iterations of the exchange algorithm.

TABLE 3

\begin{tabular}{rccc}
\multicolumn{4}{c}{ Approximation of $f(x)=-1 / \Gamma(x-3)$} \\
\hline$n$ & $\min _{1 \leq i \leq n+2}\left|E_{0}\left(x_{i}\right)\right|$ & $\left\|E_{0}\right\|$ & $\left\|f-F\left(A^{*}, x\right)\right\|$ \\
\hline 6 & $4.8 \cdot 10^{-4}$ & $8.3 \cdot 10^{-4}$ & $6.67 \cdot 10^{-4}$ \\
8 & $1.2 \cdot 10^{-4}$ & $3.4 \cdot 10^{-4}$ & $2.02 \cdot 10^{-4}$ \\
10 & $3.3 \cdot 10^{-5}$ & $6.6 \cdot 10^{-5}$ & $4.92 \cdot 10^{-5}$ \\
12 & $0.9 \cdot 10^{-5}$ & $2.1 \cdot 10^{-5}$ & $1.48 \cdot 10^{-5}$ \\
\hline
\end{tabular}

6. Acknowledgement. This paper is based in part on the author's Doctoral thesis at the Oxford University Computing Laboratory. The guidance and encouragement of Dr. D. F. Mayers is gratefully acknowledged.

Department of Mathematics

The University of Manchester

Manchester, M13 9PL, England 
1. K. APPEL, "Rational approximation of decay-type functions," Nordisk Tidskr. Informationsbehandling, v. 2, 1962, pp. 69-75. 1966.

2. D. C. Handscomb, Methods of Numerical Approximation, Pergamon Press, London, 3. C. Hastings, Approximations for Digital Computers, Princeton Univ. Press, Princeton,
N. J., 1955. MR 16, 963 .

4. G. MeINARdus, Approximation of Functions: Theory and Numerical Methods, Springer, Berlin, 1964; English transl., Springer Tracts in Natural Philosophy, vol. 13, Springer-Verlag, New York, 1967. MR 31 \# 547; MR 36 \#571.

5. JoHn R. RICE, The Approximation of Functions. Vol. I: Linear Theory, AddisonWesley, Reading, Mass., 1964. MR 29 \# 3795.

6. J. Williams, Some Numerical Problems in Theoretical Physics, Doctoral Thesis, University of Oxford, Oxford, 1968. 